Promoción de una alimentación saludable desde un enfoque de trabajo intersectorial y comunitario: experiencia de extensión universitaria en la Facultad de Ciencias Veterinarias.

Artículo de García Cachau, M.; Ferrero, V.; Villagran, M.; Millahueque, Y.

CIENCIA VETERINARIA, Vol. 21, № 1, enero-junio de 2019, ISSN 1515-1883 (impreso) E-ISSN 1853-8495 (en línea), pp. 93-102

DOI: http://dx.doi.org/10.19137/cienvet-201921107

\title{
Promoción de una alimentación saludable desde un enfoque de trabajo intersectorial y comunitario: experiencia de extensión universitaria en la Facultad de Ciencias Veterinarias
}

\author{
García Cachau, M. ; Ferrero, $V^{2}$; Villagran, M. $^{2}$; Millahueque, $Y^{1}$ \\ ${ }^{1}$ Facultad de Ciencias Veterinarias UNLPam. Calle 5 esq. 116- General Pico, La Pampa. \\ Argentina \\ ${ }^{2}$ Área Programática Hospital Gobernador Centeno, General Pico, La Pampa
}

Correo electrónico: mgarciacachau@gmail.com

\section{RESUMEN}

Se plantearon propuestas de comunicación y educación para la salud desde un enfoque de trabajo intersectorial y de la promoción de la salud. Integraron el proyecto de extensión universitaria docentes y estudiantes de la Facultad de Ciencias Veterinarias UNLPam y equipo del área programática del Hospital Gobernador Centeno, General Pico, La Pampa. El objetivo fue promover una alimentación saludable y prácticas seguras de manipulación de alimentos. Se participó en espacios intersectoriales convocados por Centros de Salud (CS) Brown y Rucci, se identificaron necesidades y planificaron intervenciones vinculadas con la alimentación saludable. Las reuniones intersectoriales fueron convocadas por los CS y participaron referentes de distintas instituciones del área programática de cada CS. Se realizaron talleres en diferentes ámbitos: establecimientos educativos de nivel inicial y primario, centros de desarrollo infantil, merenderos, salas de espera, centros de formación profesional, etc. Estas actividades permitieron identificar casos de vulnerabilidad, aclarar dudas y seguimiento por parte del equipo de salud. Se generó un espacio de diálogo y reflexión relacionados con alimentación saludable y prevención de enfermedades transmitidas por alimentos. Fue una posibilidad de articular las 
tareas de docencia, investigación y extensión. Fueron oportunidades de enseñanza, aprendizaje y acercamiento a la práctica profesional.

Palabras clave: promoción de la salud; alimentación; intersectorialidad; extensión universitaria

El trabajo intersectorial y desde la promoción de la salud es una oportunidad para que la Universidad desempeñe su Compromiso Social. Asimismo, permite la integración de las funciones de docencia, extensión e investigación.

El proyecto de extensión Cuidando nuestra alimentación para una vida saludable, aprobado por Consejo Directivo de la FCV (Res CD- FCV № 124/2016), se basó en la participación en reuniones intersectoriales convocadas por el Centro de Salud (CS) Brown de General Pico (La Pampa) ${ }^{1}$. Se pudieron identificar, planificar, implementar y evaluar distintas intervenciones vinculadas con la alimentación saludable. Se trabajó desde un enfoque de la promoción de la salud, que implicó considerar diferentes dimensiones de análisis como accesibilidad, disponibilidad, nutrición, calidad, inocuidad, tradiciones y cultura alimentaria.

La propuesta de extensión universitaria estuvo integrada por docentes y estudiantes de la carrera Medicina Veterinaria, equipo de salud del CS Brown y del Área Programática del Hospital Gobernador Centeno. Se conformó un equipo integrado por médicos veterinarios, nutricionista, trabajadoras sociales, médico, agentes sanitarios y estudiantes que permitieron abordar la temática de la alimentación desde la complejidad. Cada una de las disciplinas pudo incorporar el discurso y los saberes de la otra, con lo cual los intercambios que se generaron resultaron mucho más enriquecedores. ${ }^{(1)}$

El objetivo fue promover una alimentación saludable y prácticas seguras de manipulación de alimentos en los hogares del área. Se plantearon propuestas de comunicación y educación para la salud desde un enfoque de trabajo intersectorial y de participación comunitaria.

\section{LA EXTENSIÓN UNIVERSITARIA DESDE UN ENFOQUE INTER- SECTORIAL EN PROMOCIÓN DE LA SALUD}

La propuesta de extensión se planteó desde el trabajo intersectorial y de la promoción de la salud. La promoción de la salud es un proceso social y político que implica acciones dirigidas a modificar

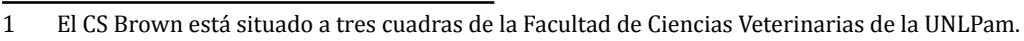


las condiciones sociales, ambientales y económicas para mitigar su impacto en la salud pública e individual. ${ }^{(2)}$ La promoción de la salud comprende información, educación, formación, comunicación, investigación, legislación, coordinación de políticas y desarrollo comunitario. (3) Sus pilares son la intersectorialidad y la participación social.

El trabajo intersectorial implica la coordinación de acciones y actividades por parte de instituciones representativas de distintos sectores sociales, mediante las intervenciones conjuntas destinadas a transformar la situación de salud, aportar al bienestar y a la calidad de vida de la población. ${ }^{(4)}$ Permite que distintos actores sociales, organizaciones e instituciones intercambien, articulen conocimientos, esfuerzos y experiencias para solucionar problemas locales. Se participa en el diagnóstico, planificación, ejecución y evaluación de las acciones implementadas.

A partir del año 2008, docentes y estudiantes de la Facultad de Ciencias Veterinarias de la UNLpam, comenzaron a participar de las reuniones intersectoriales convocada por el CS Brown. Se comenzó a trabajar en conjunto con representantes de establecimientos educativos, organizaciones barriales, organismos municipales y provinciales que están ubicados en el área programática del CS. El trabajo en red, permitió la planificación e implementación de distintas actividades a partir de las necesidades y/ o problemáticas detectadas. Las reuniones intersectoriales, son espacios de participación comunitaria, que tienen una larga historia de interacción y trabajo local participativo en la ciudad de General Pico. En tal sentido, se acuerda con Tommasino y Rodríguez (2011) ${ }^{(5)}$ la articulación de actores sociales y universitarios, con "miradas interdisciplinarias", permite la "construcción intersectorial, interinstitucional de propuestas que resuelvan problemáticas concretas" (p.26). A lo largo de estos años de participación en espacios intersectoriales se pudieron reconocer necesidades, problemáticas y percepciones de la comunidad en distintas temáticas vinculadas con la salud ambiental.

En este camino se fueron construyendo lazos y vínculos que permitieron conocernos y comunicarnos con distintos actores sociales. Se pudieron detectar problemáticas sociales, ambientales y se generaron proyectos de extensión e investigación que se vincularon con la docencia universitaria. Se comparte la idea de Cecchi, Pérez y Sanllorenti $(2013)^{(6)}$ que formar parte de espacios ya 'consolidados' de trabajo en territorio, permite visibilizar necesidades, demandas y recursos para abordar distintas problemáticas.

En estos años de participación en el espacio intersectorial del CS Brown, se observaron y detectaron distintas problemáticas 
relacionadas con la manipulación de alimentos en el ámbito doméstico y escolar. Se generó un proyecto de investigación que evidenció prácticas de manipulación de alimentos que podían implicar un peligro para la salud de niños, y niñas, adultos mayores, embarazadas, entre otros grupos de riesgo ${ }^{(7)}$

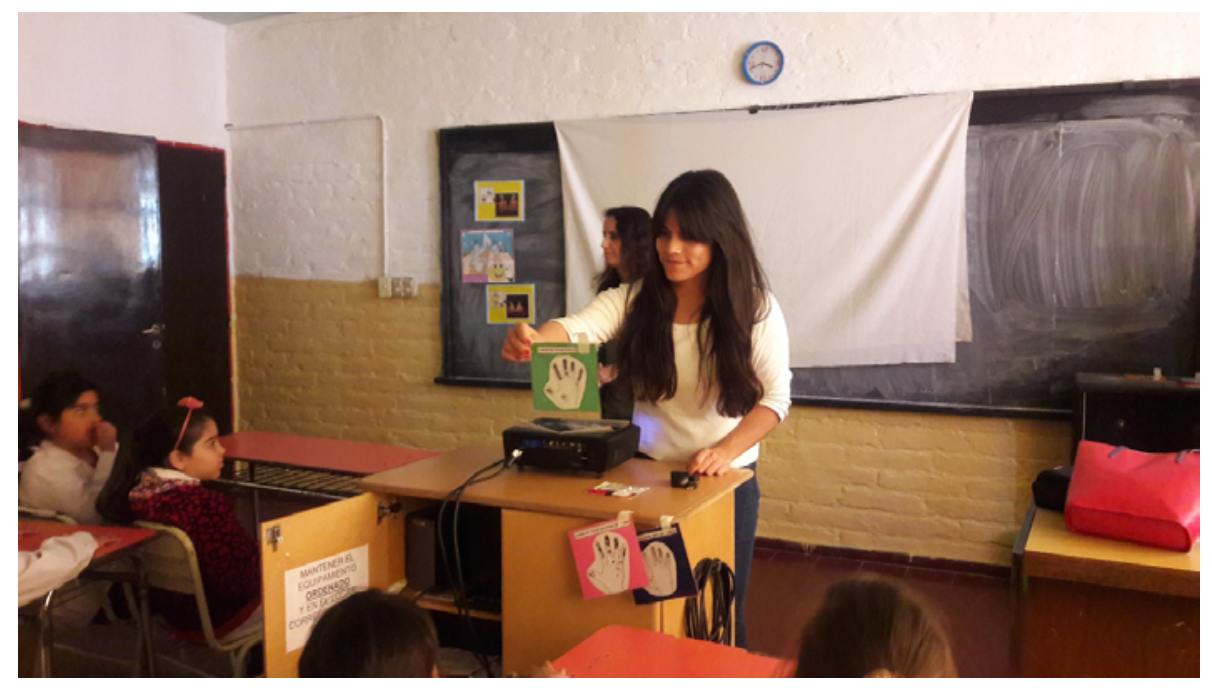

Desde el CS se detectaron en las consultas, problemáticas como sobrepeso, obesidad, diabetes, casos de diarreas, etc. Asimismo representantes de instituciones que participaban de este espacio intersectorial, expresaron preocupación por problemáticas vinculadas a la alimentación y expresaron interés en trabajar esta temática. A partir de esta situación se decidió presentar el proyecto de extensión Cuidando nuestra alimentación para una vida saludable en la FCV-UNLPam.

Es importante considerar, que la promoción de una alimentación saludable, requiere un abordaje multidimensional, es decir, no puede ser abordada desde una mirada disciplinar. En palabras de Tommasino y Rodríguez (2011) ${ }^{(5)}$ "la realidad es indisciplinada" (p.38), por lo que requiere para su transformación intervenciones interdisciplinarias. Como se mencionó anteriormente, en el caso concreto de la alimentación, se entrecruzan dimensiones económicas, productivas, tecnológicas, nutricionales, ambientales, biológicas, educativas, sociales, culturales, entre otras. Esta complejidad invita a un abordaje interdisciplinario para poder analizarlas. El espacio intersectorial es un escenario propicio para trabajar la problemática seleccionada, porque participan distintos sectores y actores sociales con diferentes formaciones, experiencias y saberes. En este contexto circulan conocimientos 
científicos de distintas disciplinas, saberes populares, creencias y tradiciones. En relación a lo antes mencionado, es importante considerar la noción de ecología de saberes propuesta por Boaventura de Sousa Santos $(2007)^{(8)}$ que, plantea la necesidad de favorecer diálogos entre los conocimientos científicos, los saberes populares y las tradicionales, que circulan en la sociedad. Promueve una nueva convivencia de saberes, primando el supuesto, que todos los saberes se enriquecen de ese diálogo.

\section{ACTIVIDADES DE EXTENSIÓN UNIVERSITARIA PROPUESTAS DESDE EL TRABAJO INTERSECTORIAL Y DE LA PROMOCIÓN DE LA SALUD}

Las actividades de extensión se planificaron e implementaron a partir de la participación en los espacios intersectoriales convocados por el Centro de Salud (CS) Brown. Con este proyecto se intentó generar un espacio de diálogo y reflexión relacionados con alimentación saludable y la prevención de enfermedades de transmisión alimentaria.

Se implementaron estrategias de comunicación y educación para la salud desde un enfoque intersectorial. Las mismas se organizaron en conjunto con los participantes del espacio intersectorial.

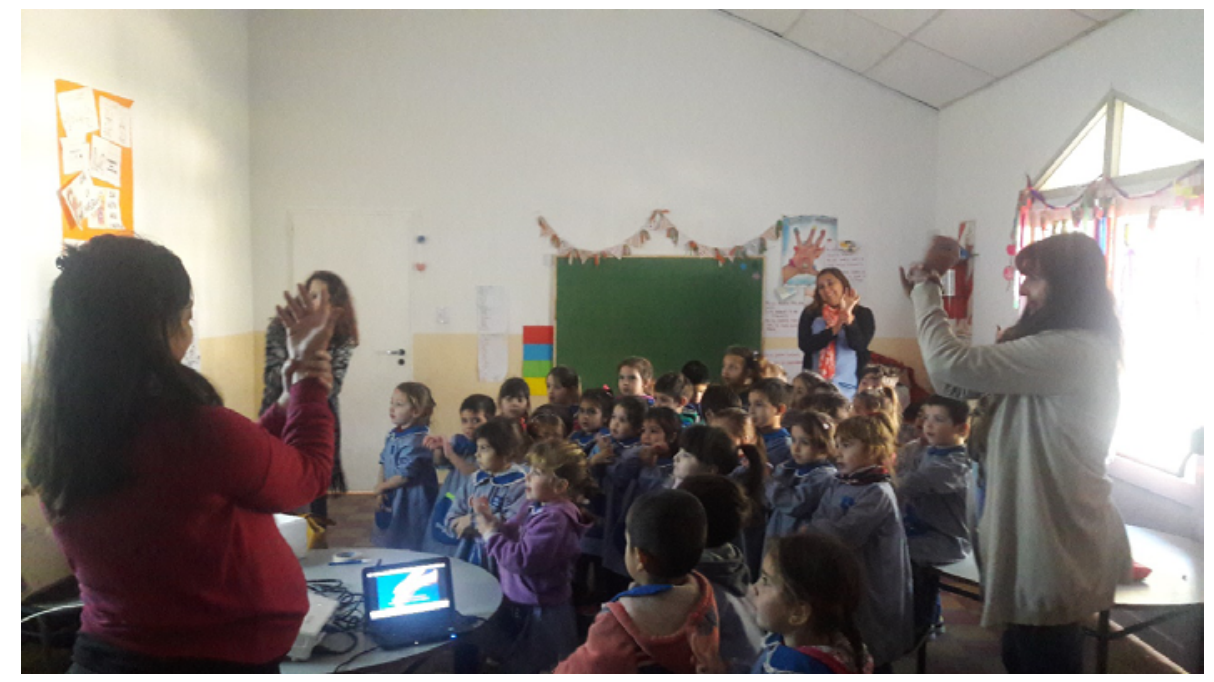

La modalidad de trabajo fueron los talleres, que se desarrollaron en diversos espacios: instituciones educativas, salas de espera, biblioteca, talleres de formación profesional, merenderos, centros de desarrollo infantil etc. A partir de imágenes, cuentos, canciones, situaciones 
cotidianas y preguntas se proponía que los participantes expresaran sus ideas y creencias en torno a la alimentación. Lejos de la tradicional forma de enseñanza y aprendizaje, esta manera de trabajo, intentó generar un espacio de comunicación no formal, se promovió la circulación de la palabra, el diálogo entre saberes y las prácticas vinculadas con la alimentación.

Los principales destinatarios fueron niños y niñas que asistían a espacios de educación formal y no formal del área, porque estaban en una etapa de construcción de hábitos vinculados con la alimentación. El trabajo con adultos permitió identificar casos de vulnerabilidad, aclarar dudas y seguimiento por parte del equipo de salud.

Se conformó un equipo de trabajo interdisciplinario e intersectorial que permitió abordar la temática de la alimentación desde la complejidad, teniendo en cuenta aspectos sociales, culturales, económicos, nutricionales y sanitarios.

Se realizaron reuniones periódicas del grupo de trabajo de planificación, ejecución y evaluación del proyecto.

Se diseñó y elaboró material didáctico y de comunicación para la salud, que fueron entregados en las distintas instituciones.

Se comenzó a visibilizar la importancia de una alimentación saludable y buenas prácticas de manipulación de alimentos en el hogar y en las escuelas.

A partir de la participación en intersectoriales y la implementación de talleres se pudieron identificar casos de vulnerabilidad, riesgo de contraer enfermedades de transmisión alimentario y otras problemáticas ambientales que requirieron el trabajo en conjunto.

A partir del interés generado en estas temáticas, se comenzó a participar en agosto de 2018 del espacio intersectorial convocadas por el CS Rucci.

Durante el año 2018, los estudiantes que optaron por la orientación en Medicina Preventiva, Salud Pública y Bromatología de la carrera Medicina Veterinaria, hicieron las prácticas de las asignaturas Vigilancia ambiental y Educación sanitaria y comunicación social, en actividades propuestas en el marco del proyecto de extensión "Cuidando nuestra alimentación para una vida saludable". Participaron en intersectoriales de los centros de salud (CS) Brown y Rucci. Seleccionaron un tema, planificaron, implementaron y evaluaron talleres en salas de espera. Fueron acompañados por docentes de la FCV y por los equipo de salud de los CS.

En el marco del Proyecto antes mencionado, se organizó en septiembre de 2018, la Jornada Salud y ambiente: experiencias de trabajo intersectorial a nivel local (Res. CD FCV 213/18). Se generó un espacio 
de encuentro y de intercambio entre estudiantes de la orientación Medicina Preventiva, Salud Pública y Bromatología, equipos de trabajo municipales, de salud pública y extensionistas de la FCV que habían realizado diferentes actividades en conjunto. Se compartieron experiencias y se señaló la necesidad de continuar generando intervenciones desde el enfoque intersectorial.

Los estudiantes que cursaron la orientación Medicina Preventiva, Salud Pública y Bromatología, evaluaron como positivo la participación en actividades propuestas en el marco de este proyecto de extensión, valoraron la posibilidad de participar en la implementación de talleres en salas de espera y en prácticas en escenarios reales para su propia formación.

\section{A MODO DE CONCLUSIÓN}

Este proyecto de extensión permitió conformar un equipo de trabajo integrado por médicos veterinarios, nutricionista, trabajadoras sociales, médico, promotoras de salud y estudiantes que permitieron abordar la temática de la alimentación desde la complejidad. Es decir, teniendo en cuenta las dimensiones sociales, culturales, económicas, nutricionales y sanitarias. Durante la planificación, la implementación y la evaluación de los de las actividades previstas, cada una de las disciplinas fue incorporando el discurso y los saberes de la otra, con lo cual el intercambio que se fue produciendo fue más enriquecedor.

Se generaron oportunidades de enseñanza, aprendizaje y acercamiento a la práctica profesional. Se convirtió en una manera de vinculación con una realidad que muchas veces es desconocida por la comunidad universitaria. Los procesos de enseñanza y aprendizaje en territorio, permitieron 'enfrentarse' con problemas reales y la complejidad que estos presentan. Este tipo de experiencias posibilitaron que los estudiantes y docentes, conozcan y se involucren con la realidad local, se pudieron relacionar contenidos teóricos abordados en distintas asignaturas, buscar respuestas a nuevas preguntas, reflexionar y cuestionar las propias prácticas. Además, este tipo de propuestas, promovieron que los estudiantes sean protagonistas de sus aprendizajes con el acompañamiento del equipo de trabajo de este proyecto, docentes, equipo de salud e integrantes del espacio intersectorial. 


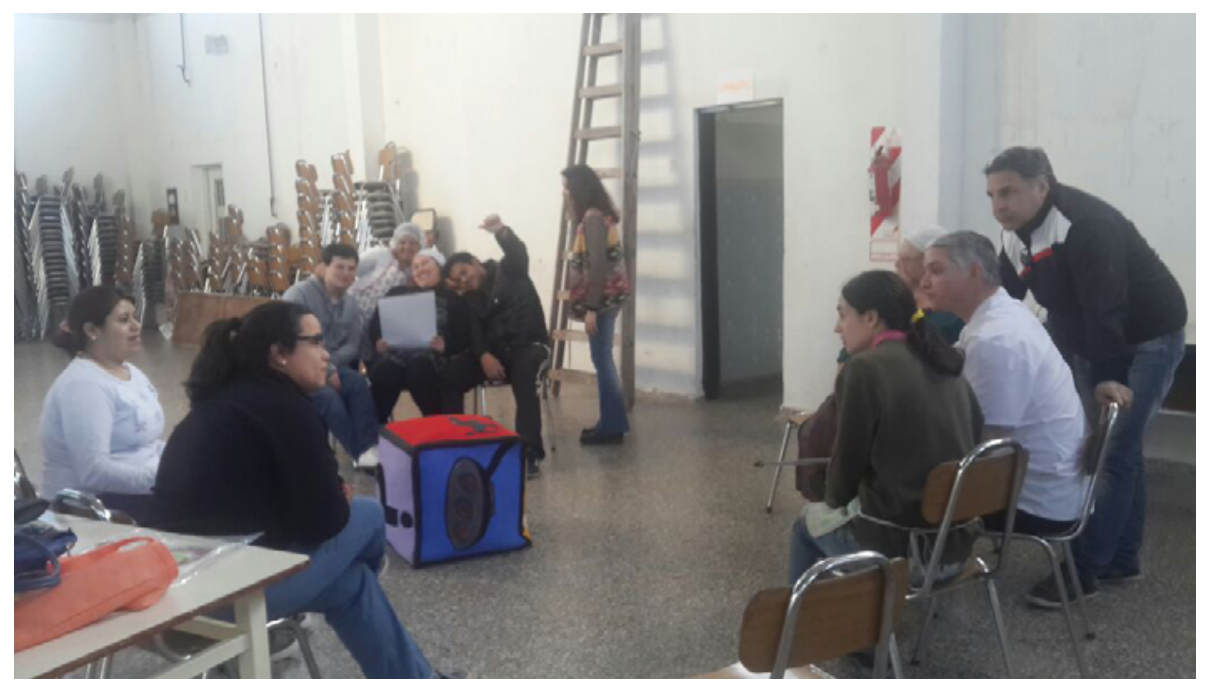

Se acuerda con Tommasino y Rodríguez (2011) (5) "la extensión concebida como un proceso dialógico contribuye a orientar la investigación y la enseñanza" (p.39). Es decir, esta concepción dialógica y transformadora de la extensión y su vinculación con la docencia y la investigación cuestiona los modos de enseñar, de aprender, de concebir y generar conocimiento en la universidad.

El abordaje de problemas reales propicia el trabajo interdisciplinario e intersectorial, permite un acercamiento al pensamiento complejo que se requiere para ejercer las distintas profesiones.

A modo de conclusión final, los espacios intersectoriales, como ámbitos de participación de distintos actores sociales ya consolidados en la ciudad, son oportunidades para pensar propuestas de extensión donde puedan sumarse docentes, estudiantes y graduados, abarcando distintas temáticas vinculadas con la Medicina Veterinaria. Esto permitirá avanzar en la integración de la docencia, extensión e investigación con Compromiso Social Universitario. El gran desafío es continuar construyendo redes de trabajo que nos enriquecen como institución al vincularlos con la comunidad de la que somos parte. 


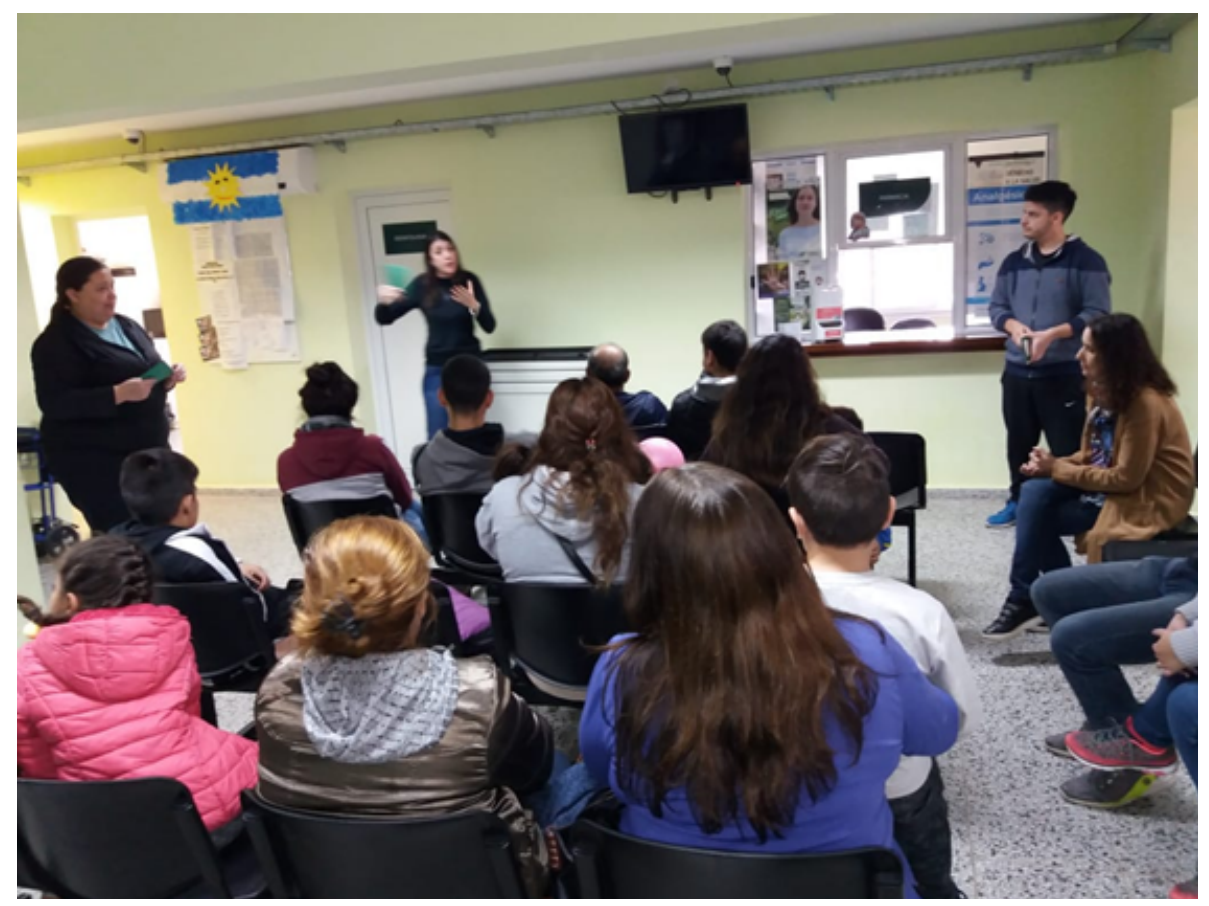




\section{Bibliografía}

1. García Cachau, M.; Ferrero, V.; Villagran, M.; Gino, L.; Millahueque, Y.; Cuellas, F.; García R.; Biegler, V.; Sartor, S.; Bistolfi, S.; Covella, M. Cuidando nuestra alimentación para una vida saludable desde un enfoque de trabajo intersectorial. Revista de la VII Jornadas de Extensión Universitaria y XI Jornadas de Investigación relacionadas con la salud y ciencias afines. Unión Latinoamericana de Extensión Universitaria. Pp. 56-58. 2018 http://www.uleu.org/uleu wp/2018/09/05/ya-esta-disponible-la-revista-de-las-xi-jornadas-de-investigacion-y-vii-jornadas-de-extension-en-salud/

2. OMS Carta de Ottawa para la Promoción de la Salud. OMS 1986. Recuperado de: http://www1.paho.org/spanish/HPP/OttawaCharterSp.pdf

3. Galvez, R. e Irazola, J. El árbol de la transformación. Manual de metodologías participativas para agentes de salud comunitaria. Buenos Aires, Argentina: CEIBA Salud y Desarrollo, Medicus Mundi y Médicos del mundo Argentina. 2006.

4. OPS Planificación local participativa. Metodologías para la Promoción de la Salud en América Latina y el Caribe. Washington, Estados Unidos: OPS. 1999. Recuperado de: http://iris.paho.org/xmlui/handle/123456789/3136

5. Tommasino, H. y Rodríguez, N. Tres tesis básicas sobre la extensión y pràcticas integrales en la Universidad de la República En: Arocena, Tomamasino, Rodríguez, Satz, Álvarez, Romano Integralidad: tensiones y perspectivas. Cuadernos de Extensión (pp. 19-42) Montevideo, Uruguay: Universidad de la República. 2011.

6. Cecchi, N.; Pérez, D. y Sanllorenti, P. Compromiso Social Universitario. De la Universidad posible a la Universidad Necesaria. Buenos Aires, Argentina: IEC-CONADU. 2013).

7. García Cachau M.; Cavagión L.; Larrieu E. Las prácticas de manipulación de alimentos en los hogares del área del Centro de Salud Brown, General Pico, La Pampa. Rev. Ciencia Veterinaria, Facultad de Ciencias Veterinarias, UNLPam, Vol.14 № 12012 ISSN 1515-1883. Pp. 19-26.

8. De Sousa Santos, B. La universidad en el siglo XXI. Para una reforma democrática y emancipadora de la universidad. Buenos Aires: Miño y Dávila-LPP. 2007. 